\title{
Control loops design in a grid supporting mode inverter connected to a microgrid
}

\author{
Giovanni M. Foglia ${ }^{(1)}$, Luisa Frosio ${ }^{(2)}$, Matteo F. Iacchetti ${ }^{(3)}$, Roberto Perini ${ }^{(1)}$ \\ ${ }^{(1)}$ DEPT OF ENERGY - POLITECNICO DI MILANO \\ via La Masa 3420156 Milano Italy Tel.: +39 / 02 - 23998506. \\ E-Mail: roberto.perini@polimi.it URL: http://www.polimi.it \\ ${ }^{(2)}$ MCM ENERGY LAB srl, via Grazzini 14 - 20158 Milano Italy \\ (3) SCHOOL OF ELECTRICAL AND ELECTRONIC ENGINEERING, UNIVERSITY OF \\ MANCHESTER, Sackville Street Building, Manchester (UK)
}

\section{Keywords}

« Microgrids », « Droop control », « Control loop », « System stability »

\begin{abstract}
This paper deals with some design aspects of the control loops in a droop-controlled VSI connected to other inverters of greater ratings or to a strong grid, which sets the frequency. The design of a damping resistor in series with the AC filter capacitor is discussed and the impact of strong feedback between the output current and the capacitor voltage is investigated. Moreover an analysis of the derivative droop coefficients is carried out through a simplified equivalent circuit.
\end{abstract}

\section{Introduction}

Microgrids with local control of voltage and frequency through inverter in grid supporting mode are becoming more and more important. Several topologies have been presented in literature [1]: essentially, they can involve two or more inverters either in parallel on the same common bar or connected through R-L lines and feeding close and remote loads. In spite of such a variety of topologies, some control issues of inverters forming a microgrid and operating in supporting grid mode can be studied by considering the basic structure in Fig. 1 .

With a strong grid, a resistor $R_{d}$ is connected in series to the capacitor [2], to avoid parallel resonance. The inductor $L_{t}-R_{t}$ represents the adaptive transformer and the line.

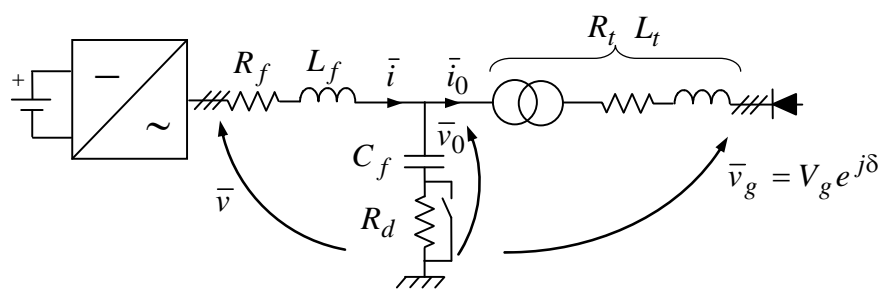

Fig. 1. Scheme of the analyzed electrical system. In case of a strong grid, the resistance $R_{d}$ is inserted to avoid parallel resonance.

A method to share the real and reactive powers between the sources consists in the droop control: derivative terms can be added to improve the stability [3]. Nevertheless, the design of the $\bar{v}_{0}$ voltage controller is not obvious, especially when the inverter is connected either to another inverter of much greater rating, which is operating in a quasi no - load condition, or to a strong grid in a grid supporting mode. In such conditions, the natural damping in the physical system is very low and a carefully designed damping resistor series connected to the capacitor is required to avoid overvoltage oscillations and damage of the inverter. Moreover, as it will be shown, the strong grid introduces a quite significant feedback between the current $\bar{i}_{0}$ and the terminal voltage $\bar{v}_{0}$ in the small signal loop 
model. As the feed-forward terms cannot totally compensate the output current $\bar{i}_{0}$, this effect should be accounted for in the voltage controller design. These topics are addressed in this paper, which analyzes the design of the damping resistor and the effect of the strong grid on the stability of the voltage loop. Finally, the design of the derivative terms of the droop control will be examined. The system in Fig. 1 can be represented by the following equations, expressed in per unit (p.u.) in a $d q$ frame with the $\mathrm{d}$ axis aligned with the reference voltage $\bar{v}_{0}^{\text {ref }}$, where $p=d / d t$ is the time derivative and $\omega_{b}$ the reference angular frequency, equal to the rated one $\omega_{n}$ :

LCL filter equations

$$
\begin{aligned}
& \bar{v}=\bar{v}_{0}+R_{f} \bar{i}+\left(L_{f} / \omega_{b}\right) p \bar{i}+j \omega L_{f} \bar{i} \\
& \left(C_{f} / \omega_{b}\right) p \bar{v}_{0}+j \omega C_{f} \bar{v}_{0}=\left(1+j \omega R_{d} C_{f}\right)\left(\bar{i}-\bar{i}_{0}\right)+\left(R_{d} C_{f} / \omega_{b}\right)\left(p \bar{i}-p \bar{i}_{0}\right) \\
& \bar{v}_{0}=V_{g} e^{j \delta}+R_{t} \bar{i}_{0}+\left(L_{t} / \omega_{b}\right) p \bar{i}_{0}+j \omega L_{t} \bar{i}_{0}
\end{aligned}
$$

Droop equations with the linear $m$ and $n$ and derivative $m_{d}$ and $n_{d}$ parameters. The average real $P$ and reactive $Q$ powers are obtained by a first order filter, with time constant $T_{p}$. $\omega^{*}$ and $V^{*}$ are the no-load values:

$$
\begin{gathered}
\omega=\left\lfloor\omega^{*}-\left(m+m_{d} p\right) P\right]=\omega^{*}-\left(m+m_{d} p\right)\left(v_{0 d} i_{0 d}+v_{0 q} i_{0 q}\right) /\left(1+p T_{p}\right) \\
v_{o d}^{r e f}=\left\lfloor V^{*}-\left(n+n_{d} p\right) Q\right\rfloor=V^{*}-\left(n+n_{d} p\right)\left(v_{0 q} i_{0 d}-v_{0 d} i_{0 q}\right) /\left(1+p T_{p}\right)
\end{gathered}
$$

Current and voltage controllers, including some compensating terms through the gains $H_{i}$ and $H_{v}$

$$
\begin{aligned}
& \bar{v}^{r e f}=\left(K_{p I}+K_{i I} / p\right)\left(\bar{i}^{r e f}-\bar{i}\right)+\bar{v}_{0}+j \omega L_{f} \bar{i} \\
& \bar{i}^{r e f}=\left(K_{p V}+K_{i V} / p\right)\left(\bar{v}_{0}^{r e f}-\bar{v}_{0}\right)+\left(H_{i} \bar{i}_{0}+H_{v} j \omega C_{f} \bar{v}_{0}\right)
\end{aligned}
$$

Power filters and load angle $\delta$ ( $\omega_{g}$ is the grid frequency)

$$
\begin{gathered}
\bar{v}=\bar{v}^{r e f} /\left(1+p T_{i n v}\right) \\
p \delta=\omega_{b}\left(\omega_{g}-\omega\right)
\end{gathered}
$$

A block diagram of the equation system is reported in Fig. 2, where the Laplace variable $s$ replaces the operator $p$. Two time constants are introduced: $T_{f}=L_{f} /\left(\omega_{b} R_{f}\right), T_{t}=L_{t} /\left(\omega_{b} R_{t}\right)$.

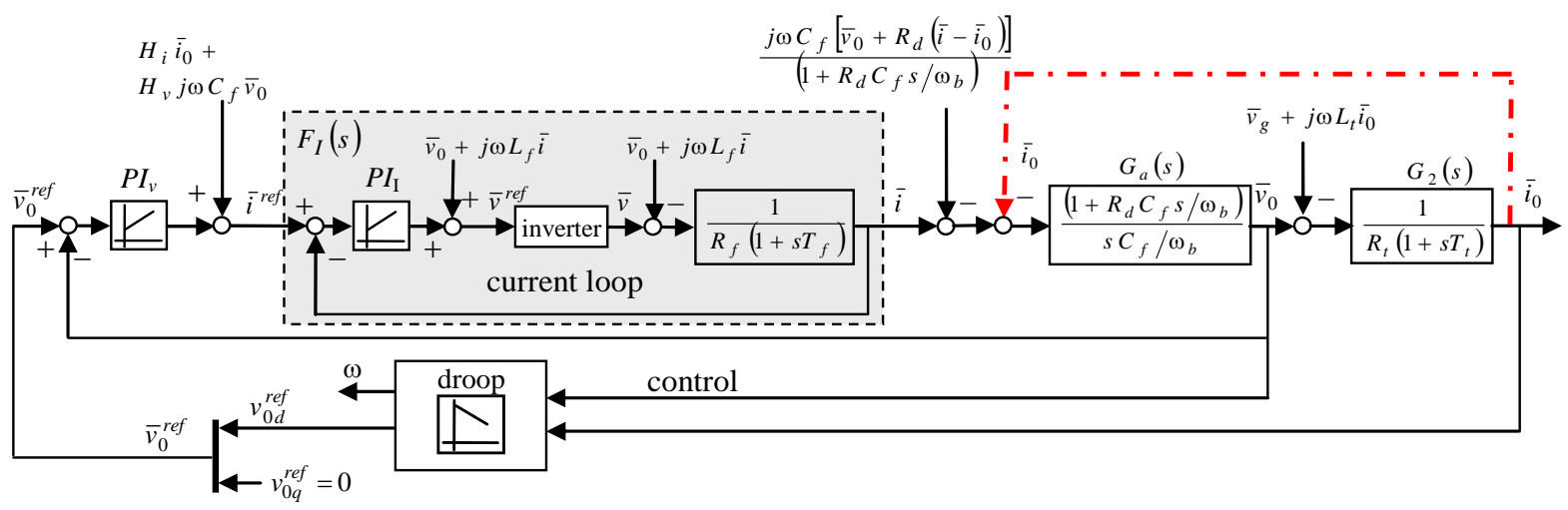

Fig. 2. Block diagram of the whole system, in the $d q$ frame. 
A design criterion for the LCL filter is reported in [2] and is based on the limitation of the series voltage drop (here 6\%) and on the capacitive reactive power due to the capacitors (5\%). According to this, the main data of the system under study are reported in Table I.

TABLE I: System Parameters

\begin{tabular}{c|c}
\hline Rated $V_{n}[\mathrm{~V}], I_{n}[\mathrm{~A}], A_{n}[\mathrm{kVA}], \omega_{n}[\mathrm{rad} / \mathrm{s}]$ & $200 ; 7 ; 2.4 ; 2 \pi 50$ \\
\hline Inverter max real $P_{\max }$ and reactive $Q_{\max }$ power & $A_{n} ; 0.6 A_{n}$ \\
\hline$R_{f}, L_{f}$ [p.u.] & $0.0022 ; 0.045$ \\
\hline$R_{d}, C_{f}$ [p.u.] & $0.61 ; 0.052$ \\
\hline$R_{t} ; L_{t}$ [p.u.] & $0.014 ; 0.016$ \\
\hline Droop coefficients $m, n$ [p.u.] & $0.01 ; 0.017$ \\
\hline Grid ang. freq. $\omega_{g}$, voltage $V_{g}$ [p.u.] & $1.000 ; 1.00$ \\
\hline Current loop $\omega_{c I}$, voltage loop $\omega_{c V}$ cross - over & $2000 ; 200$ \\
frequency [rad/s] & 0.10 \\
\hline Time constant $T_{p}$ droop power filter [s] &
\end{tabular}

\section{Choice of the damping resistor $\boldsymbol{R}_{d}$.}

In case of a strong grid (parameters $L_{t}, R_{t}$ small), a parallel resonance between the grid and the filter capacitor may arise, that can be avoided through a resistance $R_{d}$ in series to the capacitor $C_{f}$ [2]. By some manipulations on the LCL filter equations (2) and (3), the following relation can be obtained:

$$
\left[a_{0}(s)+j a_{1}(s)\right] \bar{v}_{0}=\left[a_{2}(s)+j a_{3}(s)\right] \bar{i}+\left[a_{4}(s)+j a_{5}(s)\right] \bar{v}_{g} ;
$$

Neglecting the cross-coupling terms (indicated by the imaginary $j$ ), the important terms are:

$$
\begin{aligned}
& a_{0}(s)=L_{t} C_{f}\left(s / \omega_{b}\right)^{2}+\left(R_{t}+R_{d}\right) C_{f}\left(s / \omega_{b}\right)+\left(1-\omega^{2} L_{t} C_{f}\right) ; \\
& a_{2}(s)=R_{d} L_{t} C_{f}\left(s / \omega_{b}\right)^{2}+\left(L_{t}+R_{t} R_{d} C_{f}\right)\left(s / \omega_{b}\right)+\left(R_{t}-\omega^{2} R_{d} L_{t} C_{f}\right) ; \\
& a_{4}(s)=1+\left(R_{d} C_{f}\right)\left(s / \omega_{b}\right) ;
\end{aligned}
$$

Assume that no compensating term is introduced into the voltage loop, i.e. $H_{v}=H_{i}=0$ and neglect the droop feedback. Thus, the voltage loop in Fig. 2 changes as in Fig. 3, where the internal current loop is represented by its closed loop transfer function $F_{I}(s)$.

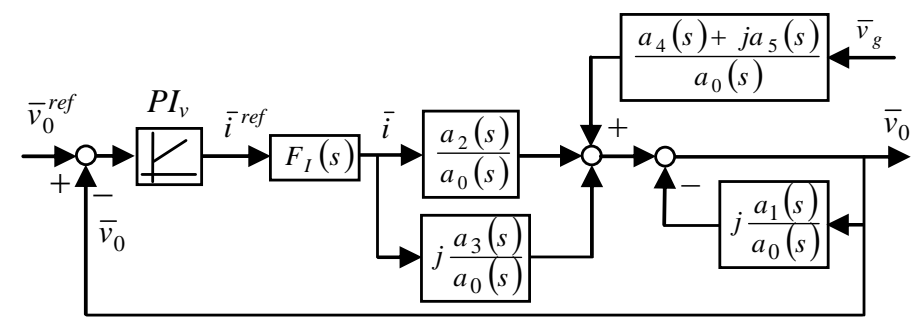

Fig. 3. Voltage loop in case of no compensation: $H_{v}=H_{i}=0 . F_{I}(s)$ is the current closed loop transfer function.

Let us analyze the term $a_{2}(s) / a_{0}(s)$ : some remarks follow for the design of the resistance $R_{d}$. First, the damping factor of the denominator should be high: 


$$
\xi_{d}=\frac{\left(R_{d}+R_{t}\right) C_{f}}{2 \sqrt{L_{t} C_{f}\left(1-\omega^{2} L_{t} C_{f}\right)}} \approx \frac{\left(R_{d}+R_{t}\right) C_{f}}{2 \sqrt{L_{t} C_{f}}}
$$

Assuming $\xi_{d}>\xi_{d}^{*}$ yields:

$$
R_{d}>2 \sqrt{L_{t} / C_{f}} \xi_{d}^{*}-R_{t}
$$

where $\xi_{d}{ }^{*}$ is the desired damping threshold (e.g.: $\xi_{d}{ }^{*}>0.5 \Rightarrow R_{d}>0.54$ p.u.).

Another limitation derives from the numerator $a_{2}(s)$, in order to avoid a zero in the right half plane, that may have negative consequences on the phase margin of the voltage loop. Thus, from the Descartes' rule on polynomials, the coefficients of $a_{2}(s)$ must have the same sign:

$$
\left(R_{t}-\omega^{2} R_{d} L_{t} C_{f}\right)>0 \Rightarrow R_{d}<\frac{R_{t}}{\omega^{2} L_{t} C_{f}}
$$

(e.g.: $\omega=1$ p.u.; $R_{d}<16.8$ p.u.)

\section{Effect of the strong grid on the voltage controller design}

The design of the current controller presents no difficulties and can be carried out according to the usual rules. In order to design the voltage controller keeping its output excursion bounded, two compensating terms can be added to the voltage loop: $H_{i} \bar{i}_{0}$ and $H_{v} j \omega C_{f} \bar{v}_{0}$ (Fig. 2). Additionally, it is useful to introduce an approximation. The design of the resistance $R_{d}$ implies that even at high frequency the voltage drop across $R_{d}$ is less than the voltage $\bar{v}_{0}[2]: R_{d}\left(\bar{i}-\bar{i}_{0}\right)<<\bar{v}_{0}$; then:

$$
\left(j \omega C_{f}\right) R_{d}\left(\bar{i}-\bar{i}_{0}\right)<j \omega C_{f} \bar{v}_{0} .
$$

Equation (2) simplifies and the transfer function between current $\bar{i}$ and voltage $\bar{v}_{0}$ is reported in the block diagram of Fig. 4 in this form:

$$
\left(C_{f} / \omega_{b}\right) p \bar{v}_{0}+j \omega C_{f} \bar{v}_{0}=\left(1+p R_{d} C_{f} / \omega_{b}\right)\left(\bar{i}-\bar{i}_{0}\right) .
$$

Because of the low value of the capacitance $C_{f}$ (in the considered case $C_{f} \approx 0.052$ p.u.), the compensating term $H_{v} j \omega C_{f} \bar{v}_{0}$ can be neglected (it corresponds to $H_{v}=0$ ). This means that the crosscoupling between $d$ - and $q$-axis is very weak.

On the other hand, the coefficient $H_{i}$ is very important. It cannot completely compensate the "disturbance" $-\bar{i}_{0}$ in the system, because $H_{i}$ is just a pure gain and, moreover, the closed loop transfer function $F_{I}(s)$ of the current loop is not perfectly known. Thus if the grid is strong, the feedback $G_{2}(s)$ must be taken into account in the design of the voltage controller, even if the feed-forward term $H_{i} \bar{i}_{0}$ is introduced. Some steps are necessary to pass from the block diagram in Fig. 2 to a more useful diagram as in Fig. 4-c: they are represented in Fig. 4-a and 4-b . Here $F_{I}(s)$ is approximated by a first order system:

$$
F_{I}(s)=\bar{i}(s) / \bar{i}^{r e f}(s) \approx \mu_{I}\left(1+s / \omega_{c I}\right)^{-1}=\mu_{I}\left(1+s T_{c I}\right)^{-1}
$$

The feedback becomes (Fig. 4-c):

$$
H_{a}(s) \approx\left(\frac{1-H_{i} \mu_{I}}{R_{t}}\right) \frac{\left(1+s T_{c I} /\left(1-H_{i} \mu_{I}\right)\right)}{\left(1+s T_{c I}\right)\left(1+s T_{t}\right)}
$$


Since $\mu_{I}$ is not precisely known (but $\mu_{I} \approx 1$ ), $H_{i}$ must be chosen less than one with a certain margin, to avoid a positive feedback that takes to instability. A low value of the line resistance $R_{t}$ implies a high value of the gain, even if $H_{i} \mu_{I} \approx 1$. Thus, the feedback $H_{a}(s)$ (20) cannot be neglected in the design of the voltage controller. Fig. 5 shows the transfer function $F_{a}(s)=G_{a}(s) /\left(1+G_{a}(s) H_{a}(s)\right)$ as a function of $H_{i}$. It can be seen that, even when $H_{i}$ approaches one, the feedback $H_{a}(s)$ has a significant impact on $F_{a}(s)$ and must be taken into account in the design of the voltage control.
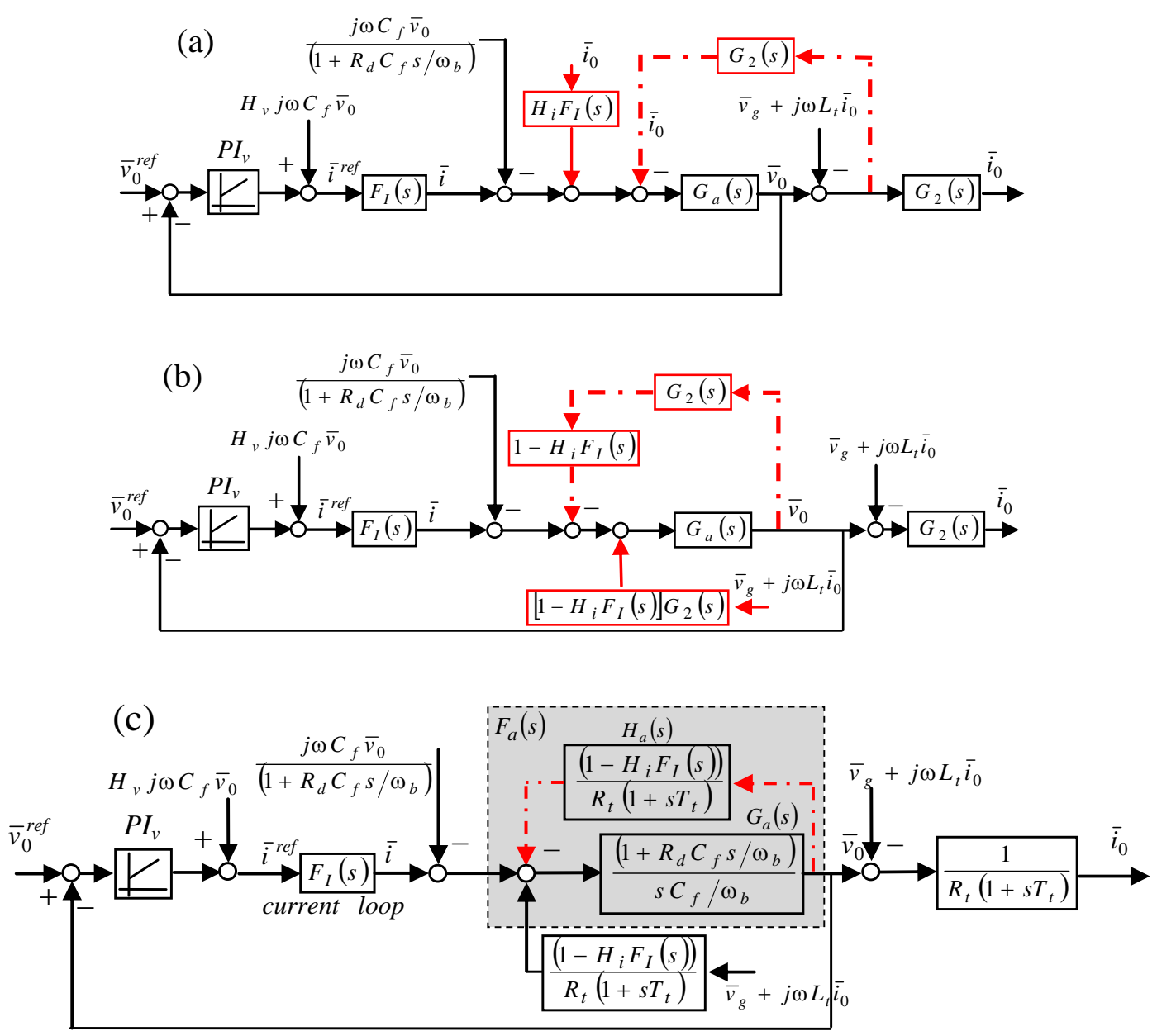

Fig.4 Deduction of a scheme of the voltage loop for the design purpose. (a), (b): preliminary manipulations starting from Fig. 2. (c): final scheme. The compensating term $H_{i} \bar{i}_{0}$ has been inserted and taken after $F_{I}(s)$. In case of a strong grid $\left(R_{t}, L_{t}\right.$ small), the feedback of the line current $\bar{i}_{0}$ must be considered, because it cannot be thoroughly compensated by $H_{i} \bar{i}_{0}$. 


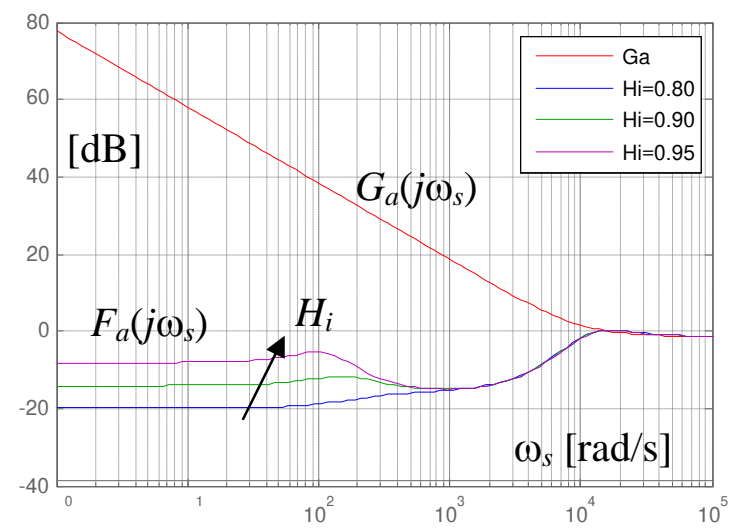

Fig. 5. Transfer functions $F_{a}\left(j \omega_{s}\right)$ of the process to be taken into account for the design of the voltage regulator. Refer to Fig. 4 . The curves differ for the parameter $H_{i}$.

The compensating term $H_{i} \bar{i}_{0}$ can reduce the excursion of the voltage controller, but it has no effect on the actuator, that is on the rating of the inverter.

\section{Influence of the droop parameters on the stability}

The system shows the dominant eigenvalues with a small damping factor and sometimes in the right half plane. In order to preserve the stability of the system, the derivative terms $m_{d}$ and $n_{d}$ are often inserted in the droop equations (3), (4) [3]. A method to design them and to analytically study their effect is here reported.

The starting point is the deduction of a reduced system representative of the dominant poles. Assume ideal current and voltage loops with an infinite bandwidth $\left(\bar{i}=\bar{i}^{\text {ref }}, \bar{v}_{0}=\bar{v}_{0}^{\text {ref }}\right)$. The equations which define the system are now: (3), (4)-(5), and (9). It is possible to linearize them around a stable condition (indicated by capital letters or superscript ${ }^{0}$ ), where the vector $\bar{v}_{0}$ is on the d-axis: $V_{0 d} \approx 1$ p.u.; $V_{0 q}=0$. Moreover $\delta_{0}<0, I_{0 d}>0 ; I_{0 q}<0$ since real power and inductive power flow from the inverter towards the grid. Then, the linearization gives:

$$
\begin{aligned}
& \Delta \bar{v}_{0}-j V_{g} e^{j \delta^{0}} \Delta \delta=R_{t}\left(1+p T_{t}\right) \Delta \bar{i}_{0}+j L_{t}\left(\omega^{0} \Delta \bar{i}_{0}+\bar{I}_{0} \Delta \omega\right) \\
& \Delta \omega=\Delta \omega^{*}-\frac{m\left(1+\tau_{d m} p\right)}{1+T_{p} p}\left(\Delta v_{0 d} I_{0 d}+V_{0 d} \Delta i_{0 d}+\Delta v_{0 q} I_{0 q}\right) \\
& \Delta v_{o d}^{r e f}=\Delta V^{*}-\frac{n\left(1+\tau_{d n} p\right)}{1+T_{p} p}\left(\Delta v_{0 q} I_{0 d}-\Delta v_{0 d} I_{0 q}-V_{0 d} \Delta i_{0 q}\right) \\
& p(\Delta \delta)=-\omega_{b} \Delta \omega
\end{aligned}
$$

where $T_{t}=L_{t} /\left(\omega_{b} R_{t}\right), \tau_{d m}=m_{d} / m, \tau_{d n}=n_{d} / n$. These equations are represented by a block diagram of transfer functions (Fig. 6), where the approximations $\Delta v_{0 q} \approx \Delta v_{0 q}{ }^{\text {ref }}=0$ and $\Delta v_{0 d} \approx \Delta v_{0 d}{ }^{\text {ref }}$ have been used. The starting diagram in Fig. 2 consists in a Multiple Input - Multiple Output (MIMO) system, with coupling terms between the axes d-q. This new diagram in Fig. 6 is instead a Single Input - Single Output (SISO) block system. Some approximations and manipulations can be adopted to simplify (21)-(24) and obtain the final block diagram in Fig. 7 which allows to analyze the stability conditions and to design the parameters $m_{d}$ and $n_{d}$. In Fig. 7, the two subsystems $F_{5}(s)$ and $F_{6}(s)$ are highlighted, and three independent SISO closed loops are derived: they link the main quantities, such as $\Delta \varepsilon_{v 0 q}$ to $\Delta v_{y}, \Delta v_{y}$ to $\Delta \omega$ and $\Delta v_{0 q}$ to $\Delta \omega$. 


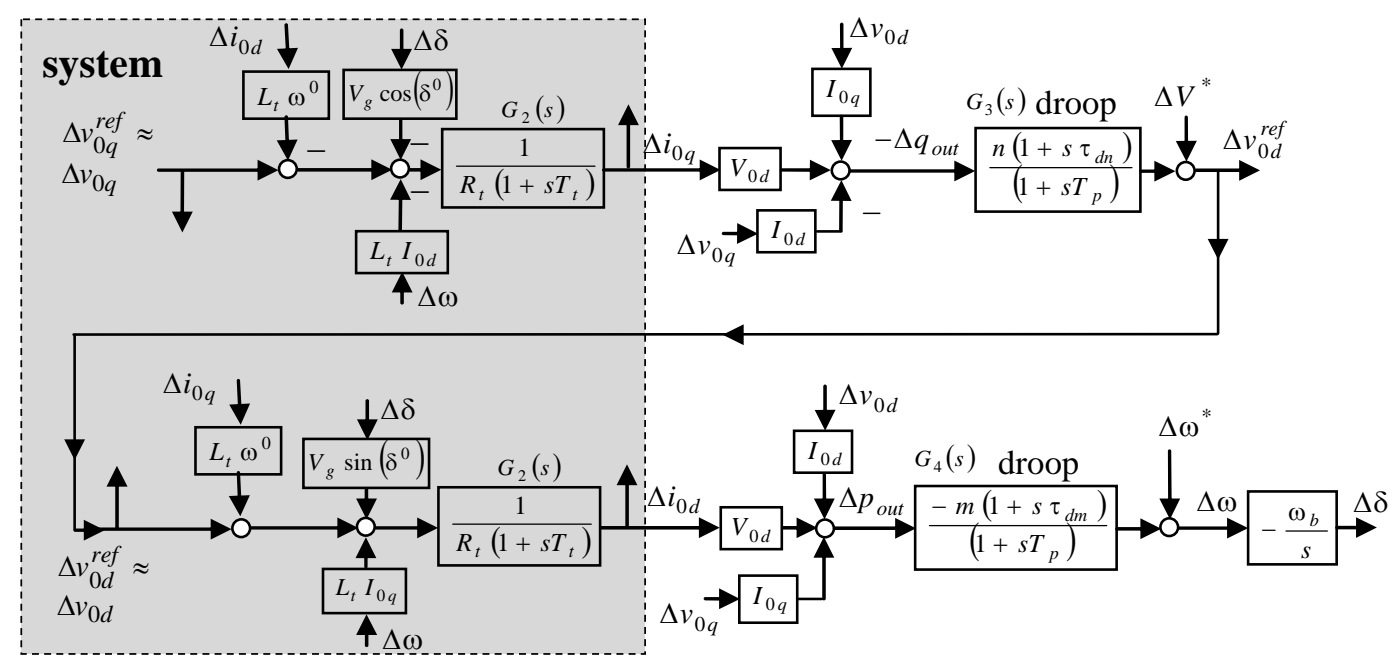

Fig. 6. Complete block diagram of the linearized system, assuming current loops with an infinite bandwidth. This is a Single Input - Single Output (SISO) block system.

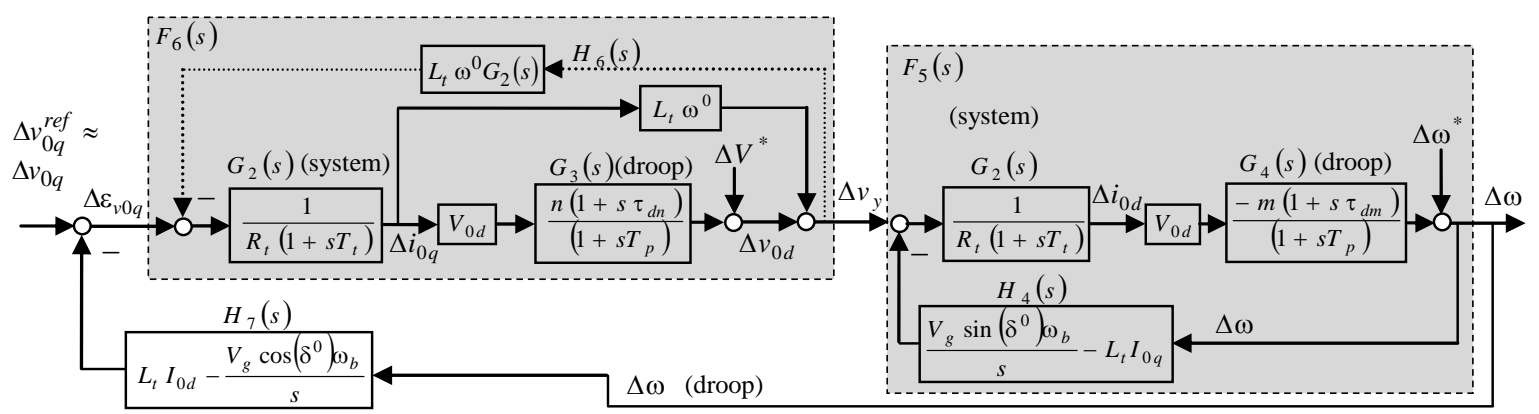

Fig. 7. New arrangement of the block diagram of Fig. 6.

This equivalent circuit is the starting point to analytically find the stability conditions of the system. It will be used hereafter to analyse the influence of the derivative droop components on the stability.

\section{Design of the Droop Derivative Coefficients $\boldsymbol{m}_{\boldsymbol{d}}$ and $\boldsymbol{n}_{\boldsymbol{d}}$.}

The block diagram in Fig. 7 represents the simplified model of the system in load operation. Some meaningful loops can be recognized: their analysis allows to discuss the effect of the droop parameters on the performance of the system. The first loop is the subsystem called $F_{5}(s)$; its open loop transfer function is:

$$
\begin{aligned}
& L_{5}(s)=G_{2}(s) G_{4}(s) H_{4}(s) V_{0 d}=\frac{V_{0 d} V_{g} m \omega_{b}\left(-\sin \left(\delta^{0}\right)\right)}{R_{t}} \frac{\left(1+s \tau_{d m}\right)\left(1-s \tau_{1}\right)}{s\left(1+s T_{t}\right)\left(1+s T_{p}\right)} \\
& \tau_{1}=\left(L_{t} I_{0 q}\right) /\left(V_{g} \omega_{b} \sin \left(\delta^{0}\right)\right)
\end{aligned}
$$

Since $\delta^{0}<0$, the gain is positive and greater than one. The zero $\tau_{1}^{-1}$ is usually at high frequencies and can be neglected in this low frequency analysis. Because $\delta^{0}$ usually has a small value, the gain of $L_{5}\left(j \omega_{s}\right)$ is low; thus the system is stable for all (reasonable) $\tau_{d m}$ values (dashed-dot line in Fig. 8) .

A second loop is the subsystem $F_{6}(s)$ : its feedback is depicted with a dotted line. The open loop transfer function is: 


$$
\begin{aligned}
& L_{6}(s)=G_{2}(s)\left(G_{3}(s) V_{0 d}+L_{t} \omega^{0}\right) H_{6}(s)=\frac{\left(V_{0 d} n+L_{t} \omega^{0}\right) L_{t} \omega^{0}}{R_{t}^{2}} \frac{\left[1+s \tau_{G 3 b}\left(\tau_{d n}\right)\right]}{\left(1+s T_{p}\right)\left(1+s T_{t}\right)^{2}} \\
& \tau_{G 3 b}\left(\tau_{d n}\right)=\left(V_{0 d} n \tau_{d n}+L_{t} \omega^{0} T_{p}\right) /\left(V_{0 d} n+L_{t} \omega^{0}\right)
\end{aligned}
$$

If $\tau_{d n}<T_{p}$ then $\tau_{d n}<\tau_{G 3 b}<T_{p}$, while if $\tau_{d n}>T_{p}$ then $T_{p}<\tau_{G 3 b}<\tau_{d n}$. Thus, this loop suggests that $\tau_{d n}$ must be lower than $T_{p}$ : in fact, when $\tau_{d n}$ approaches $T_{p}$, at the cut off frequency the slope equals -2 and reduces the phase margin (see Fig. 9). This loop suggests that $\tau_{d n}$ has a small influence on stability.

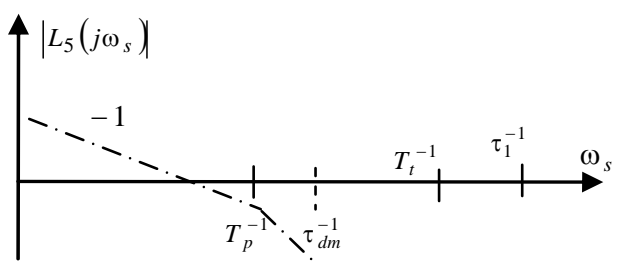

Fig. 8. Bode diagram of the open loop transfer function $L_{5}(s)$. The case where the gain of $L_{5}(s)$ is low is reported.

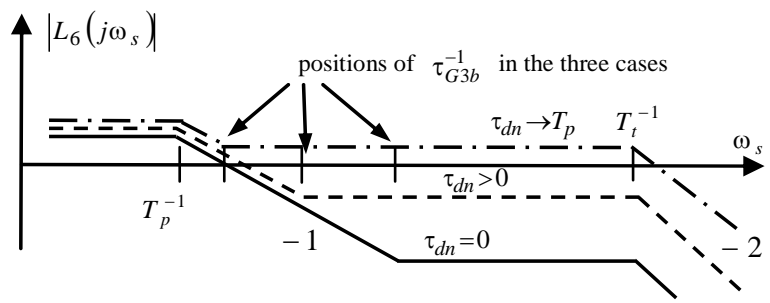

Fig. 9. Bode diagram of the open loop transfer function $L_{6}(s)$ when $n_{d}=0\left(\tau_{d n}=0\right.$, continuous line) and $n_{d} \neq 0$ ( $\tau_{d n} \neq 0$ dashed and dashed-dot lines). In the third case $\left(\tau_{d n} \rightarrow T_{p}\right)$ a less stable system derives.

In order to analyse the external loop in Fig. 7, the transfer functions $F_{5}(s)$ between $\Delta v_{y}$ and $\Delta \omega$ as well as $F_{6}(s)$ between $\Delta \varepsilon v_{0 q}$ and $\Delta v_{y}$ must be calculated. In fact, the open loop transfer function is:

$$
L_{7}(s)=F_{5}(s) F_{6}(s) H_{7}(s)
$$

An approximated expression can be found valid in the range $T_{p}^{-1}<\omega_{s}<T_{t}^{-1}$ :

$$
L_{7 a p}(s) \approx \frac{\left(V_{g} V_{0 d} m \omega_{b}\right)}{R_{t}} \frac{\left(1+s \tau_{d m}\right)}{s\left(1+s T_{t}\right)\left(1+s T_{p}\right)}
$$

The approximated open loop transfer function $L_{7 a p}(s)(30)$ and its bode diagram in Fig. 10 show the importance of the parameter $\tau_{d m}$ in order to get the stability: $\tau_{d m}$ should be chosen less than $T_{p}$. Additionally, an increase of the gain of $L_{7 a p}(s)$ may lead to instability. Such a gain depends on the voltage squared $V_{0 d}\left(V_{0 d} \approx V_{g}\right)$, on the droop coefficient $m$ and is inversely proportional to the line resistance $R_{t}$.

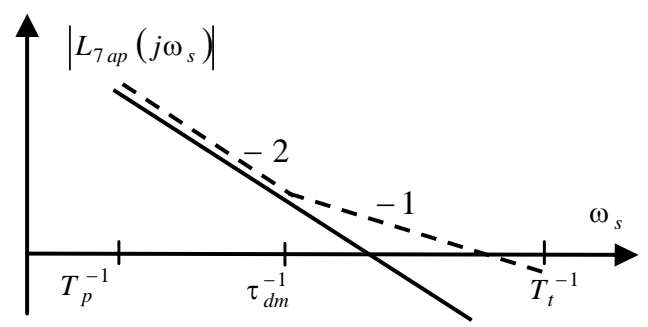

Fig. 10. Bode diagram of the transfer function $L_{7 a p}(s)$. Continuous line: $m_{d}=0$, slope -2 ; dashed line $m_{d} \neq 0$, slope -1 . The second case provides a stable system. 


\section{Numerical validation.}

Two types of validation have been carried out. The former deals with the stability analysis of the external loop, in order to check the importance of the droop derivative parameters $m_{d}$ and $n_{d}$. The latter deals with the design of the voltage controllers.

Through the complete system of equations (1) - (9), the dominant eigenvalues can be found together with their damping factor. They can be compared with the same parameters obtained by the analysis of the three open loop transfer functions $L_{5}(s), L_{6}(s), L_{7 a p}(s)$, above all from the last. It is well known that for a second order system the cut - off frequency is close to the dominant poles and that the relation between the damping factor $\xi_{d \text { ap }}$ and the phase margin $\varphi_{m}$ is $\xi_{d a p}=\sin \left(\varphi_{m} / 2\right)$. Some results as for $L_{7 a p}(s)$ are reported in Table II, for the considered experimental system with: $T_{p}^{-1}=10 \mathrm{rad} / \mathrm{s} ; T_{t}^{-1}=$ $269 \mathrm{rad} / \mathrm{s}, \mu_{\text {L7ap }}=146 \mathrm{p} . \mathrm{u}$. $=43.3 \mathrm{~dB}$ (gain of $L_{7 a p}$ ).

In case 1 with $\tau_{d m}=\tau_{d n}=0$, the results obtained by the complete and the simplified models coincide because both of them show lack of stability; a phase margin $\varphi m=3.5$ deg means instability.

An increase of $\tau_{d m}$ implies a stable system (case 2), while a $\tau_{d n}$ different from zero has a negligible effect (case 3).

The values of $m_{d}$ and $n_{d}$ depend on the actual value of $m$ and $n$ respectively and on the main time constants of the system, as $T_{p}$ and $T_{t}$.

A second check is carried out as for the design of the voltage loop controller. In fact, if we take into account the feedback $H_{a}(s)$ in Fig. 4, the parameters of the voltage controller are (data in Table I):

$K_{p V}=1.47$ p.u. $K_{i V}=596$ p.u. $/ \mathrm{s}$

If we do not take $H_{a}(s)$ into account, the same parameters are:

$K_{p V}=0.025$ p.u. $K_{i V}=1.47$ p.u./s

which imply a complete different behaviour when they are implemented into the real system.

Figs. 11 show the results from simulation after the connection of the droop controlled inverter. The reference $v_{0 d r e f}$ and the actual $v_{0 d}$ voltages are shown in two cases: the voltage controller is designed taking and not taking into account the feedback $H_{a}(s)$ in Fig. 4c, respectively. In the first case the system is even unstable.

TABLE II Dominant poles $p_{1,2}$ and damping coefficient $\xi_{d}$ from the complete model (1)(9) and cut off frequency $\omega_{c}$ and phase margin $\varphi_{m}$ from the reduced model $L_{7 a p}(s)$, as a function of the droop derivative time constants $\left(\tau_{d m}, \tau_{d n}\right) ; V_{g}=1.0$ p.u.; $V^{*}=1.02$ p.u. $\approx V_{g}$; $\omega^{*} \approx 1.009$ p.u. $; I_{0 d} \approx 0.94 ; I_{0 q} \approx-0.10$.

\begin{tabular}{|l|l|l|l|l|l|l|l|}
\hline & & & \multicolumn{3}{|c|}{$\begin{array}{c}\text { Complete model } \\
\text { (eigenvalue analysis) }\end{array}$} & \multicolumn{3}{|c|}{$\begin{array}{c}\text { Reduced model (simplified } \\
\text { analysis) }\end{array}$} \\
\hline case & $\frac{\tau_{d m}}{T_{p}}$ & $\frac{\tau_{d n}}{T_{p}}$ & $\begin{array}{c}p_{1,2} \\
{[\mathrm{rad} / \mathrm{s}]}\end{array}$ & $\begin{array}{c}\xi_{d} \\
{[\%]}\end{array}$ & $\begin{array}{c}\omega_{\mathrm{c}} \\
{[\mathrm{rad} / \mathrm{s}]}\end{array}$ & $\begin{array}{c}\varphi_{\mathrm{m}} \\
{[\mathrm{deg}]}\end{array}$ & $\begin{array}{c}\xi_{d} a \\
{[\%]}\end{array}$ \\
\hline 1 & 0 & 0 & $\begin{array}{l}1.9 \pm \mathrm{j} 34 \\
\text { unstable }\end{array}$ & -5.6 & $\begin{array}{l}34 \\
\text { unstable }\end{array}$ & 3.5 & 3 \\
\hline 2 & 0.4 & 0 & $-44 \pm \mathrm{j} 40$ & 74 & 51 & 60 & 50 \\
\hline 3 & 0.4 & 0.4 & $-50 \pm \mathrm{j} 17$ & 95 & 56 & 63 & 52 \\
\hline
\end{tabular}

\section{Conclusion}

This paper has analyzed some design aspects of the control loops in a microgrid with droop-controlled VSIs, especially when the line impedance is very small and stability problems arise. The design of a 
damping resistor in series with capacitor, necessary to avoid parallel resonance, has been studied. The impact of the strong feedback between the output current and the capacitor voltage has been analyzed: when such an effect is not taken into account in the design of the voltage controller, the system may be unstable. Additionally, the derivative droop coefficients have been designed starting from a simplified equivalent system in a dynamical operating condition.
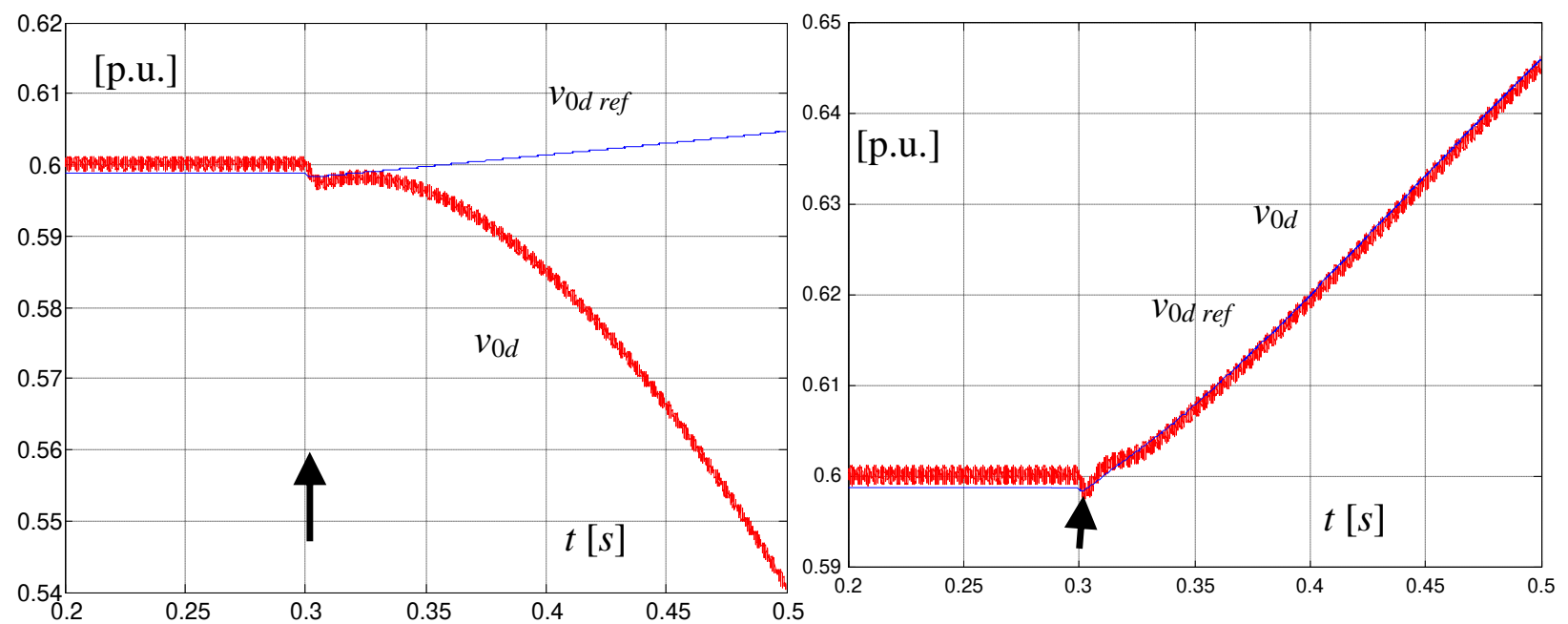

Fig. 11. Actual and reference voltages $v_{0 d}$ and $v_{0 r e f}$ after the insertion of the droop control. Left: voltage controller designed without taking into account the feedback $H_{a}(s)$ in Fig. 4c. Right: voltage controller designed taking into account the feedback $H_{a}(s)$ in Fig. 4c.

\section{References}

[1] J. Rocabert, A. Luna, F. Blaabjerg; P. Rodríguez, “Control of Power Converters in AC Microgrids” IEEE Trans. on Power Electronics, Vol 27, Issue 11, 2012, pp. 4734 - 4749.

[2] M. Liserre, F. Blaabjerg, S. Hansen, “ Design and control of an LCL-filter-based three-phase active rectifier”, IEEE Trans. on Ind. Appl., Vol. 41, No.5, Sept/Oct. 2005, pp. 1281 - 1290

[3] Y. A. I. Mohamed, E. F. El-Saadany, “Adaptive decentralized droop controller to preserve power sharing stability of parallel inverters in distributed generation microgrids”, IEEE Trans. on Power Electronics, Vol. 23, No.6, Nov. 2008

[4] J.P. Guerrero, J.C. Vasquez, J. Matas, M.Castilla, L. Garcia de Vicuna, “Control strategies for flexible microgrid based on parallel line-interactive UPS systems”, IEEE Trans. on Ind. Electronics, Vol. 56, No.3, Mar. 2009, pp. 726-735

[5] W. Yao, M. Chen, J. Matas, J.M. Guerrero, Z. Quiang, “ Design and analysis of the droop control method for parallel inverters considering the impact of the complex impedance on the power sharing”, IEEE Trans. on Ind. Electronics, Vol. 58, No.2, Feb. 2011, pp. 576-587

[6] N. Pogaku, M. Prodanovic, T. Green, “ Modeling, analysis and testing of autonomous operation of an inverter-based microgrid”, IEEE Trans. on Power Electronics, Vol. 22, No.2, Mar. 2007, pp

[7] K. De Brabandere, B. Bolsen, J. Van der Keybus, A. Woyte, J. Driesen, R. Bemans, “ A voltage and frequency droop control method for parallel inverters”, IEEE Trans. on Power. Electronics, Vol. 22, No.4, July 2007

[8] X. Yu, A.M. Khambadkone, H. Wang, S.T.S. Terence, “ Control of parallel- connected power converters for low voltage microgrid - Part I: a hybrid control architecture”, IEEE Trans. on Power Electronics, Vol. 25, No.12, Dec. 2010, pp. 1962-2970

[9] C. N. Rowe, T. J. Summers, R. E. Betz, D. J. Cornforth, T. G. Moore, “Arctan power-frequency droop for improved microgrid stability”, IEEE Trans. on Power. Electronics, Vol. 28, No.8, Aug. 2013

[10] E. Planas, A. Gil-de-Muro, J. Andreu, I. Kortabarria, I.Martinez de Alegria, “ Design and implementation of a droop control in a d-q frame for islanded microgrid”, IET Renew. Power Gener., 2013, vol. 7, Iss. 5, pp.458-474.

[11] I. J. Gabe, V. F. Montagner, H. Pinheiro "Design and implementation of a robust current controller for VSI connected to the grid through an LCL filter”, IEEE Trans. on Power. Electronics, Vol. 24, No.6, June 2009 